\title{
GuideLine-ADHERENT INITIAL INTRAVENOUS ANTIBIOTIC THERAPY for Hospital-AcQuired/Ventilator-associated Pneumonia is Clinically Superior, Saves Lives and is Cheaper than Non GUIDELINE ADHERENT THERAPY
}

\author{
M. H. Wilke'1, R. F. Grube'1, K. F. Bodmann² \\ ${ }^{1}$ Dr. Wilke GmbH - inspiring.health, Munich, Germany \\ ${ }^{2} \mathrm{Head}$ of Internal Intensive Care Unit and interdisciplinary emergency medicine, Klinikum Barnim - Werner Forssmann hospital, \\ Eberswalde, Germany
}

\begin{abstract}
Introduction: Hospital-acquired pneumonia (HAP) often occurring as ventilator-associated pneumonia (VAP) is the most frequent hospital infection in intensive care units (ICU). Early adequate antimicrobial therapy is an essential determinant of clinical outcome. Organisations like the German PEG or ATS/ IDSA provide guidelines for the initial calculated treatment in the absence of pathogen identification. We conducted a retrospective chart review for patients with HAP/VAP and assessed whether the initial intravenous antibiotic therapy (IIAT) was adequate according to the PEG guidelines

Materials and Methods: We collected data from 5 tertiary care hospitals. Electronic data filtering identified 895 patients with potential HAP/VAP. After chart review we finally identified 221 patients meeting the definition of HAP/VAP. Primary study endpoints were clinical improvement, survival and length of stay. Secondary endpoints included duration of mechanical ventilation, total costs, costs incurred on the intensive care unit (ICU), costs incurred on general wards and drug costs.

Results: We found that 107 patients received adequate initial intravenous antibiotic therapy (IIAT) vs. 114 with inadequate IIAT according to the PEG guidelines. Baseline characteristics of both groups revealed no significant differences and good comparability. Clinical improvement was $64 \%$ over all patients and $82 \%(85 / 104)$ in the subpopulation with adequate IIAT while only $47 \%(48 / 103)$ inadequately treated patients improved $(\mathrm{p}<0.001)$. The odds ratio of therapeutic success with GA versus NGA treatment was 5.821 ( $p<0.001$, [95\% CI: 2.712-12.497]). Survival was $80 \%$ for the total population $(\mathrm{n}=221), 86 \%$ in the adequately treated (92/107) and 74\% in the inadequately treated subpopulation $(84 / 114)(p=0.021)$. The odds ratio of mortality for GA vs. NGA treatment was 0.565 ( $\mathrm{p}=0.117$, [95\% CI: 0.276-1.155]). Adequately treated patients had a significantly shorter length of stay (LOS) (23.9 vs. 28.3 days; $p=0.022$ ), require significantly less hours of mechanical ventilation (175 vs. $274 ; \mathrm{p}=0.001$ ), incurred lower total costs (EUR 28,033 vs. EUR 36,139, p = 0.006) and lower ICU-related costs (EUR 13,308 vs. EUR 18,666, p = 0.003).
\end{abstract}

Drug costs for the hospital stay were also lower (EUR 4,069 vs. EUR 4,833) yet not significant. The most frequent types of inadequate therapy were monotherapy instead of combination therapy, wrong type of penicillin and wrong type of cephalosporin.

Discussion: These findings are consistent with those from other studies analyzing the impact of guideline adherence on survival rates, clinical success, LOS and costs. However, inadequately treated patients had a higher complicated pathogen risk score (CPRS) compared to those who received adequate therapy. This shows that therapy based on local experiences may be sufficient for patients with low CPRS but inadequate for those with high CPRS. Linear regression models showed that single items of the CPRS like extrapulmonary organ failure or late onset had no significant influence on the results.

Conclusion: Guideline-adherent initial intravenous antibiotic therapy is clinically superior, saves lives and is less expensive than non guideline adherent therapy. Using a CPRS score can be a useful tool to determine the right choice of initial intravenous antibiotic therapy. The net effect on the German healthcare system per year is estimated at up to 2,042 lives and EUR $125,819,000$ saved if guideline-adherent initial therapy for HAP/VAP were established in all German ICUs.

\section{List of abbreviations:}

ALOS = Average length of stay in a given DRG, basis for determining whether a patient causes more costs than reimbursement;

LOS = Length of stay in hospital;

HAP $=$ Hospital acquired pneumonia;

VAP $=$ Ventilator-associated pneumonia;

$\mathrm{CAP}=$ Community acquired pneumonia;

DRG $=$ Diagnoses related groups, systems to classify patients based on their resource consumptions;

$\mathrm{CW}=$ Cost-weight of a given DRG, directly related to clinical and economical "severity" (resource consumption);

CPRS $=$ Complicated pathogen risk score;

ICD = International Classification of Diseases;

ICD-10 = ICD, Revision 10;

PDX = Primary diagnosis (in ICD-10);

SDX $=$ Secondary diagnosis (in ICD-10);

$\mathrm{HMV}=$ Hours of mechanical ventilation;

ICU $=$ Intensive care unit; 
ICU days = Treatment days on an intensive care unit; MRSA = Methicillin-resistant staphylococcus aureus;

CI = Clinical improvement;

PEG = Paul-Ehrlich Gesellschaft, providing guidelines on antibiotic therapy;

ATS = American Thoracic society;

BLI = Beta-lactamase inhibitor;

LRTI = Lower respiratory tract infection;

IIAT $=$ Initial intravenous antibiotic therapy;

$\mathrm{GA}=$ IIAT according to guideline = guideline adherent;

NGA = IIAT not according to guideline = non-guideline adherent

\section{INTRODUCTION}

As a growing problem in patient care, hospital-acquired infections require particular attention in intensive care patients since they are significantly associated with worse outcomes [1] and higher costs [2-4]. Pneumonia (LRTI) is the most prevalent infection among ICU-patients $[5,6,7]$. The incidence of VAP ranges between 9.3 and 13.6 cases per 1,000 ventilation days. Early adequate antimicrobial therapy is an essential determinant of clinical outcome. Choosing the appropriate agent, however, remains challenging since in most cases no data on the identity and susceptibility of the pathogen is available at the time of treatment initiation [8-12].

Several guidelines on the initial therapy of HAP and VAP were developed in the last years. Recently, updated versions of the recommendations issued by the ATS/IDSA (USA) and PEG (Germany) were published [13-15]. These guidelines acknowledge the risk of difficult-to-treat pathogens as causative agents of nosocomial pneumonia. The recommended regimens include monotherapy with acylaminopenicillines + beta-lactamase inhibitors, 3rd generation cephalosporins, quinolones, carbapenemes and various combination regimens.

So far only a few published studies investigated the impact of clinician adherence to therapeutic guidelines on clinical outcomes or at least the usefulness of guidelines in clinical care [16-18]. We investigated the effects of guideline adherence on clinical and economical outcomes of patients with HAP / VAP in the ICU.

\section{Materials And Methods}

German hospitals collect and submit vast amounts of administrative, clinical data. A subset of approximately 250 hospitals additionally provides cost data. These data are primarily used for the annual evaluation and cost calculation within the German DRG-System (GDRG). These datasets are frequently used in clinicaleconomical analyses as they include all primary and secondary diagnoses (ICD-10 encoded) [19-24].

We investigated HAP/VAP outcomes and therefore focussed on ICD codes representing pneumonia as a secondary diagnosis. In our study we collected the above-specified data from 5 hospitals (2 university hospitals and 3 major urban tertiary hospitals). These hospitals were selected because we assumed that guidelines adherence was highest in tertiary care and university hospitals. Moreover the selected hospitals participate in the German DRG annual evaluation program. The German Institute for the Hospitals Remuneration System (InEK) that performs these evaluations uses high quality standards regarding cost data.

Together with clinical advisors we selected the patient population of interest. As HAP/VAP are specifically relevant on ICU $[5,9,25]$, we used the recommendations of the PEG guidelines for adults to determine whether a given initial intravenous antibiotic therapy (IIAT) was adherent to guidelines. Thus we only included patients who where at least 18 years old at the time of treatment initiation.

Identifying patients for clinical economic analyses from DRG-related data has certain limitations and therefore needs special attention. The diagnoses are encoded in ICD-10 GM (German modification). A patient has a primary diagnosis (PDX) which is the retrospectively identified cause for hospitalization. Each dataset also contains a number of secondary diagnoses (SDX). A SDX must be documented if the disease caused significant resource consumption during the hospital stay. Some SDXs, the so-called complications and comorbidities (CC), are cost-relevant within the DRG system. In the German DRG dataset an SDX has no date and no flag whether it is already present on admission (POA) or not. Thus the mere existence of a pneumonia code among the SDXs does not unequivocally indicate a HAP/VAP. However, since 2009/10 the ICD-10 GM includes the code U69.00 as an indicator SDX for HAP/VAP, making HAP/VAP identification much easier.

In our dataset we excluded all patients with pneumonia or exacerbated COPD as PDX. All patients having a pneumonia code as SDX and no such code as PDX were included.

The PEG guidelines (2004/2005 version) for IIAT of HAP are similar to the ATS or IDSA guidelines in terms of the therapeutic options. We chose the PEG recommendations since they provide a convenient scoring system that defines three different patient groups based on the risk of the involvement of complicated pathogens. [26-31].

Table 1a provides an overview to the CPRS system in the PEG-recommendations. Table $1 \mathrm{~b}$ shows the therapy recommendations depending on the calculated score.

Table $1 a$. Risk factors associated with the involvement of complicated bacterial pathogens in HAP/VAP.

\begin{tabular}{ll}
\hline Risk factor & Score \\
\hline Age $>$ 65 yrs & 1 \\
Structural lung disease (e.g. COPD) & 2 \\
Previous antibiotic treatment & 2 \\
Late onset (5 or more days after admission) & 3 \\
Invasive or non-invasive mechanical ventilation on & 3 \\
onset date & \\
Extrapulmonary organ failure & 4 \\
\hline
\end{tabular}

The total complicated pathogen risk score (CPRS) is calculated by adding up all individual scores for risk factors identified in a given patient. 
Table 1b. PEG therapeutic recommendations for HAP/VAP depending on the complicated pathogen risk score (CPRS).

\begin{tabular}{|c|c|}
\hline Group $\mathbf{I}(\mathrm{CPRS}=1$ or 2$)$ & Aminopenicillin/BLI or cephalosporin $2 / 3$ a or quinolone $3 / 4$ or carbapenem 2 \\
\hline Group II (CPRS = 3 to 5$)$ & Acylaminopenicillin/BLI or cephalosporin $3 \mathrm{~b} / 4$ or quinolone $2 / 3$ or carbapenem 1 \\
\hline \multirow[t]{3}{*}{ Group III (CPRS = 6 and more) } & Cephalosporin 3b/4 or acylaminopenicilline/BLI or carbapenem 1 \\
\hline & Always in combination with either \\
\hline & Quinolone $2 / 3$ or aminoglycoside* \\
\hline
\end{tabular}

Using the initial data filter, we identified patients with probable HAP/VAP. We then performed the following analyses via medical chart review (if available):

- Check ICD coding and medical documentation to verify if HAP/VAP was present and patient can be included.

- Checking other inclusion/exclusion criteria like the ICU-days ( $\max 20$ days)

- Determine the HAP/VAP date of onset using radiology results, laboratory findings and clinical documentation [25]

- Calculate the CPRS according to PEG recommendations [15]

- Document IIAT starting with the date of onset.

- Compare the actual IIAT to the PEG recommendations

- Determine whether the treatment was adequate or non-adequate according to the PEG guidelines. The criterion of IIAT adequacy was used to divide the cohort in two subpopulations.

- Check 'clinical improvement' as documented by laboratory results, radiology or clinical/nursing documentation, and respiratory parameters in mechanically ventilated patients. Clinical improvement was defined as improvement of respiratory parameters, decreasing CRP, decreasing WBC and/or radiological signs of improvement. The patient was categorized as 'improved' only if improvement occurred within 4 days of treatment initiation. Patients with unclear improvement status data were categorized as indeterminate.

- The chart review was performed by two clinical infectious disease specialists. In addition, the charts were reviewed by ICD coding experts to determine whether coding was correct.

For the patients included in the final analysis set, we gathered the data listed in Table 2 for further analysis. Table 3 shows the primary and secondary endpoints of the statistical analysis.

\section{RESULTS}

In total, we received 211,084 datasets from the 5 hospitals for the year 2007. Through the initial data filter we identified 895 patients with probable HAP/VAP. 647 patients were excluded during chart reviews. For 223 patients the medical record was missing (could not be retrieved) or not complete (e.g. laboratory or mi-
Table 2. Data collected for patients included in the final analysis set.

\section{Demographic characteristics \\ Age}

Sex

\section{Study-relevant data}

Onset-date

CPRS score

Treatment group according to PEG guidelines

Adequate IIAT according to guidelines (yes/no)

Clinical improvement

\section{Treatment-related data}

Initial diagnosis

DRG and related CW

Length of stay

Duration of mechanical ventilation

Discharge code $($ death $=$ code 7$)$

\section{Economic data}

Total costs

Costs on normal wards

Costs incurred on ICU

Total drug costs

Drug costs on ICU

Drug costs on general ward

Table 3. Primary and secondary endpoints of statistical analysis.

\begin{tabular}{lcc}
\hline Endpoint & Primary & Secondary \\
\hline Clinical improvement & $\mathrm{X}$ & \\
Survival (no discharge code 7) & $\mathrm{X}$ & \\
Length of stay in hospital & $\mathrm{X}$ & \\
Total costs (in hospital) & & $\mathrm{X}$ \\
ICU costs & & $\mathrm{X}$ \\
Duration of mechanical ventilation & & $\mathrm{X}$ \\
Total medication costs & & $\mathrm{X}$ \\
Medication costs on ICU & & $\mathrm{X}$ \\
\hline
\end{tabular}

crobiology data). 166 patients turned out to be cases with CAP. This is due to the fact that pneumonia and another relevant condition were already present on admission but the other diagnosis was chosen as primary diagnosis for DRG purposes. In most cases this was acute left-heart failure combined with pneumonia. In 


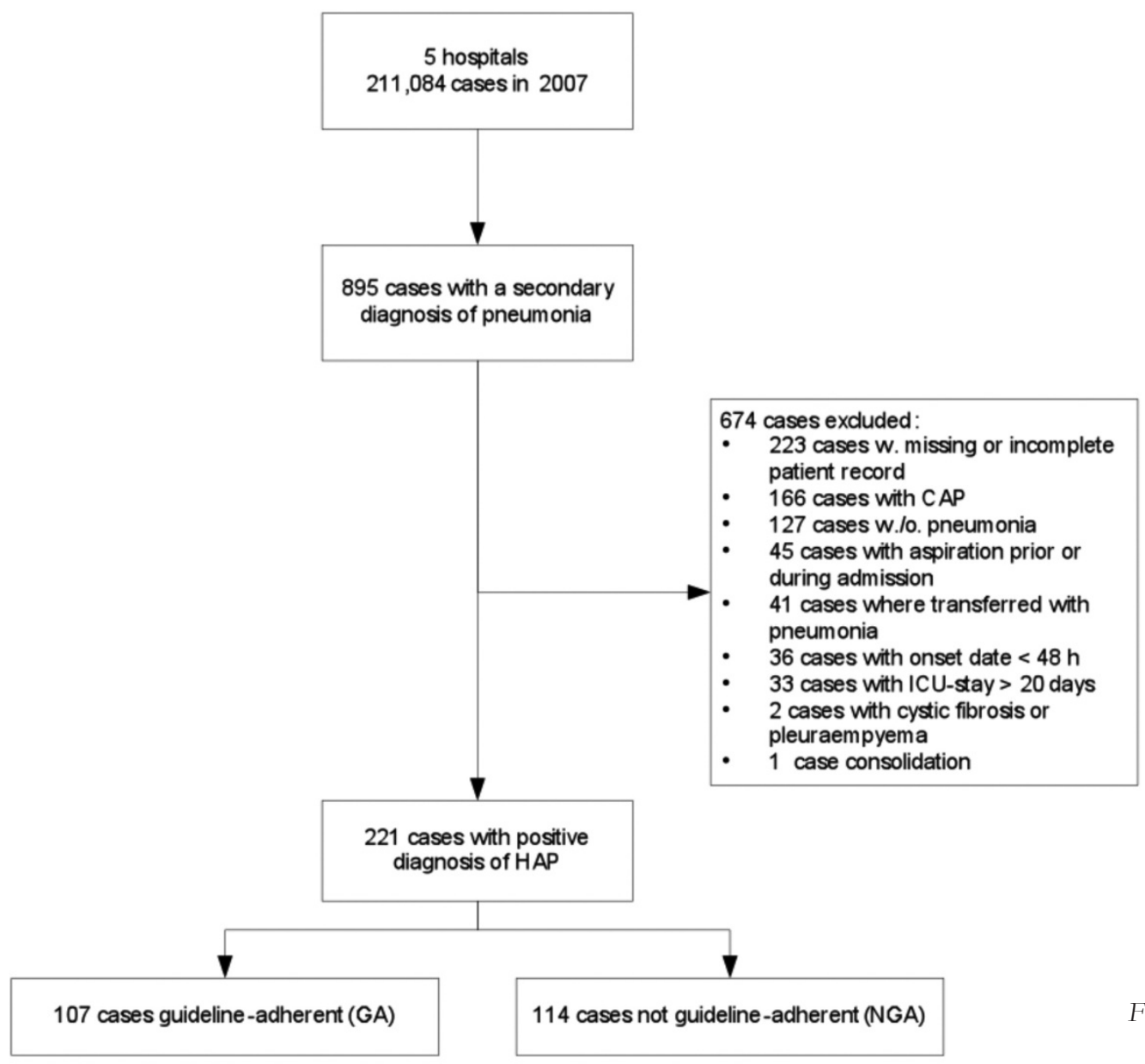

Fig. 1. Patient distribution.

127 cases, pneumonia was coded as SDX but could not be validated via chart review. 45 cases had aspiration during or shortly after admission, 41 cases had been transferred from another hospital (i.e. IIAT started there) and 36 cases had pneumonia onset earlier than 48 hours after admission. Therefore, these three groups did not meet the definition of HAP, i.e. pneumonia occurring $48 \mathrm{~h}$ or later after hospital admission [25]. 33 patients had an ICU stay of more than 20 days, 2 cases had cystic fibrosis as underlying chronic illness which is not covered by the general recommendations for HAP/VAP. Finally 1 patient was excluded since he was readmitted within less than 14 days and both stays were consolidated due to DRG regulations. The cost data therefore do not longer reflect the hospital stay in which the pneumonia occurred.

After this process, 221 patients were included in the final analysis set. Shapiro-Wilkes test of the subpopulations showed normal distribution of values in both subpopulations. $T$ tests on showed no significant differences.

Figure 1 provides an overview of the inclusion process.

Age, distribution of sex and mean CW of the patients as well as primary diagnosis and the type of intervention (surgical, interventional, medical only) were used to determine whether the two subpopulations (adequate vs. inadequate IIAT) were comparable at baseline. Shapiro-Wilkes test was used to check for normal distribution of values in both subpopulations. Student $\mathrm{T}$ test was used to check for significance of differences. Table 4 shows the baseline patient characteristics of the final analysis set. There were no statistically significant differences in patient distribution between the two subpopulations.

We then tested for significant differences in the primary endpoints clinical improvement and survival. Table 5 shows the results of the chi-square test for differences in clinical improve $\neg$ ment. The proportion of patients with improvement was significantly higher in patients with adequate therapy according to guidelines.

Table 6 shows the results of the chi-square test for differences in survival.

The association to clinical improvement and survival of possible cofactors such as age, gender, presence of neurological, cardiologic disease or polytrauma was tested by logistic regression.

The rates of clinical improvement were $64 \%$ for all patients, $82 \%(85 / 104)$ in the GA-group and $47 \%$ $(48 / 103)$ in the NGA group, respectively $(\mathrm{p}<0.001)$. The odds ratio of therapeutic success with GA versus NGA treatment was 5.821 ( $\mathrm{p}<0.001$, [95\% CI: 2.71212.497]). The odds ratio for age (elderly vs. non-elderly) was 0.983 ( $\mathrm{p}=0.215$, [95\% CI: $0.957-1.010]$ ), the OR for gender (female vs. male) was $1.030(\mathrm{p}=0.940$ [95\% CI: 0.475-2.232]) and the OR for the disease groups (present vs. not present) was $1.048(\mathrm{p}=0.902$, [95\% CI: 0.496- 2.214]).

The survival rates were $80 \%$ for all patients, $86 \%$ in the GA-group (92/107) and 74\% in the NGA-group, respectively $(0=0.021)$. The odds ratio of mortality 
Table 4. Baseline characteristics of the two subpopulations in the final analysis set.

\begin{tabular}{|c|c|c|c|c|}
\hline \multirow{2}{*}{\multicolumn{2}{|c|}{ Characteristics }} & \multicolumn{2}{|c|}{ Adequate IIAT according to PEG guidelines } & \multirow[b]{2}{*}{$\mathrm{p}$ value } \\
\hline & & yes & No & \\
\hline \multicolumn{2}{|c|}{ Number of cases } & 107 & 114 & \\
\hline \multicolumn{2}{|c|}{ Male / female } & $73 / 34$ & $73 / 41$ & \\
\hline \multicolumn{2}{|c|}{$\operatorname{Age}(+/-\mathrm{SD})$} & $63.4(+/-13.3)$ & $65.2(+/-14.0)$ & n.s. \\
\hline \multicolumn{2}{|c|}{ DRG cost weight (+/- SD) } & $8.663(+/-5.947)$ & $10.071(+/-5.882)$ & n.s. \\
\hline \multicolumn{5}{|c|}{ Type of intervention } \\
\hline \multicolumn{2}{|c|}{ Surgical procedure } & 102 & 99 & n.s. \\
\hline \multicolumn{2}{|c|}{ Other procedure } & 5 & 4 & n.s. \\
\hline \multicolumn{2}{|c|}{ Medical } & 7 & 4 & n.s. \\
\hline \multicolumn{5}{|c|}{ Primary diagnosis (ICD 10 chapter) } \\
\hline I & Infections and parasitic diseases & 1 & 2 & n.s. \\
\hline II & Neoplasms & 18 & 15 & n.s. \\
\hline III & Blood & 3 & 2 & n.s. \\
\hline VI & Nervous system & 4 & 4 & n.s. \\
\hline IX & Circulatory system & 63 & 67 & n.s. \\
\hline $\mathrm{X}$ & Respiratory system & 2 & 2 & n.s. \\
\hline $\mathrm{XI}$ & Digestive system & 4 & 2 & n.s. \\
\hline XIII & Musculoskeletal system & 2 & 1 & n.s. \\
\hline XIV & Genitourinary system & 1 & 0 & n.s. \\
\hline XVIII & $\begin{array}{l}\text { Symptoms, signs and abnormal } \\
\text { clinical and laboratory findings, } \\
\text { not classified elsewhere }\end{array}$ & 0 & 1 & n.s. \\
\hline XIX & $\begin{array}{l}\text { Injury, poisoning and other } \\
\text { consequences of external causes }\end{array}$ & 16 & 11 & n.s. \\
\hline
\end{tabular}

n.s. $=$ not statistically significant

Table 5. Clinical improvement according to IAAT adequacy.

\begin{tabular}{l|cc|c}
\hline & \multicolumn{2}{|c|}{ Adequate IIAT according to PEG guidelines } & \\
& Yes & No & p value \\
\hline $\mathbf{N}=$ & 104 & 103 & \\
Clinical improvement & 85 & 48 & $<0.001$ \\
& $(81.7 \%)$ & $(46.6 \%)$ & \\
\hline
\end{tabular}

* The table includes 207 patients. 14 patients of the final analysis set were excluded since clinical improvement was indeterminate as follow-up x-ray images or laboratory results could not be retrieved.

Table 6. Survival according to IIAT adequacy.

\begin{tabular}{l|cc|c}
\hline & \multicolumn{2}{|c|}{ Adequate IIAT according to PEG guidelines } & \\
& Yes & No & p-value \\
\hline $\mathbf{N}=$ & 107 & 114 & \\
Survived & 92 & 84 & 0.021 \\
& $(85.6 \%)$ & $(73.7 \%)$ & \\
\hline
\end{tabular}

for GA vs. NGA treatment was 0.565 ( $\mathrm{p}=0.117$, [95\% CI: 0.276-1.155]). Having neurological disease, cardiologic disease or polytrauma was found to be a strong negative predictor of survival with an OR of 0.241 ( $p=0.012$, [95\% CI: 0.096-0.609]). The odds ratios for age (elderly vs. non-elderly) and gender (fe- male vs. male) were $1.026(\mathrm{p}=0.79$, [95\% CI: 0.997 1.056]) and $1.246(p=0.545,[95 \%$ CI: 0.612-2.536]), respectively.

For the subsequent economical analyses the patients with lethal outcome were excluded. All outcome measures were compared via Student T test. 
Table 7. Economic outcomes according to IIAT adequacy (deceased patients excluded from analysis).Costs in $€$.

\begin{tabular}{|c|c|c|c|c|}
\hline \multirow[b]{2}{*}{ Means (+/-SD) } & \multicolumn{2}{|c|}{ Adequate IIAT according to PEG guidelines } & \multirow[b]{2}{*}{ Difference } & \multirow[b]{2}{*}{$\mathrm{p}$ value } \\
\hline & Yes & No & & \\
\hline $\mathbf{N}=$ & 92 & 84 & -- & -- \\
\hline Length of stay, days & $\begin{array}{c}23.9 \\
(+/-12.9)\end{array}$ & $\begin{aligned} & 28.3 \\
&(+/-12.7)\end{aligned}$ & -4.5 & 0.022 \\
\hline Duration of mechanical ventilation, hours & $\begin{array}{c}175 \\
(+/-174)\end{array}$ & $\begin{array}{c}274 \\
(+/-223)\end{array}$ & -99 & 0.001 \\
\hline \multicolumn{5}{|l|}{ Hospital costs } \\
\hline Total costs, $€$ & $\begin{aligned} & 28,033 \\
&(+/-16,574)\end{aligned}$ & $\begin{aligned} & 36,139 \\
&(+/-20,036)\end{aligned}$ & $-8,106$ & 0.006 \\
\hline Total costs ward & $\begin{array}{l}3,062 \\
(+/-3,207)\end{array}$ & $\begin{aligned} & 2,918 \\
&(+/-3,216)\end{aligned}$ & 145 & n.s. \\
\hline Total costs ICU & $\begin{array}{c}13,308 \\
(+/-10,706)\end{array}$ & $\begin{array}{l}18,666 \\
(+/-12,068)\end{array}$ & $-5,358$ & 0.003 \\
\hline \multicolumn{5}{|l|}{ Drug costs $€$} \\
\hline Total drug costs & $\begin{array}{c}4,096 \\
(+/-4,061)\end{array}$ & $\begin{array}{c}4,833 \\
(+/-5,585)\end{array}$ & -737 & n.s. \\
\hline Drug costs ward & $\begin{array}{c}655 \\
(+/-1,632)\end{array}$ & $\begin{array}{c}622 \\
(+/-1,783)\end{array}$ & 33 & n.s. \\
\hline Drug costs ICU & $\begin{array}{l}2,083 \\
(+/-2,395)\end{array}$ & $\begin{array}{l}2,372 \\
(+/-2,412)\end{array}$ & -288 & n.s. \\
\hline
\end{tabular}

Table 8. Comparison of chosen vs. recommended PEG treatment group in the subpopulation with inadequate IIAT ( $\mathrm{n}=114$ ).

\begin{tabular}{l|ccc}
\hline & \multicolumn{3}{|c}{ Recommended PEG treatment group according to CPRS score } \\
\hline Chosen therapy group & I & II & III \\
\hline Therapy does not fit in any group & 2 & 4 & 7 \\
Therapy group I & & 34 & 14 \\
Therapy group II & & 38 & 53 \\
Total & 2 & 74
\end{tabular}

Table 9. Types of IIAT inadequacy.

\begin{tabular}{lc}
\hline Type of deviation from PEG recommendation & $\mathbf{n}=$ \\
\hline Monotherapy instead of combination therapy & 64 \\
Aminopenicillin/BLI instead of & 16 \\
Acylaminopenicillin/BLI & 16 \\
Cephalosporin 3a instead of Cephalosporin 3b/4 & \\
Wrong combination therapy & 10 \\
Wrong monotherapy (chosen antibiotic not in & 4 \\
recommendations) & 3 \\
No therapy at onset date of HAP/VAP & 1 \\
Oral instead of intravenous medication & $\mathbf{1 1 4}$ \\
Total &
\end{tabular}

Table 7 shows the primary and secondary economic outcomes for the two sub $\neg$ populations (deceased patients excluded from analysis).

Patients in the subpopulation with adequate IIAT show a significantly shorter length of stay (23.9 vs.
28.3 days; $\mathrm{p}=0.022)$, have significantly less hours of mechanical ventilation (175 vs. $274 ; \mathrm{p}=0.001)$, incurred lower total costs (EUR 28,033 vs. EUR 36,139; $\mathrm{p}=0.006)$ and lower ICU costs (EUR 13,308 vs. EUR $18,666, p=0.003)$. Drug costs for the hospital stay were also lower (EUR 4,069 vs. EUR 4,833) yet not significant.

We then analyzed the specific IIAT used and ranked the type of inadequacy of IIAT.

Table 8 shows a comparison between chosen and recommended PEG therapy groups.

Table 9 shows the frequencies of different types of IIAT inadequacy.

The most frequent type of inadequacy was monotherapy in patients with a CPRS score of 6 or higher (Group III) that should receive combination therapy according to PEG recommendations. The second most common type of inadequacy was the use of aminopenicillin/BLI (e.g. amoxicillin/clavulanic acid) instead of an acylaminopenicilin/BLI (e.g. piperacilline/tazobactam) in cases having a CPRS score of 3-5 (Group II). Another common deviation from the PEG recommendation was the use of a cephalosporin 
group $2 / 3 a$ (e.g. cefazoline) instead of group $3 b / 4$ (e.g. ceftazidime) in cases having a CPRS score of 3-5 (Group II).

\section{Discussion}

The results of this study clearly show the clinical and economical superiority of adequate initial antibiotic treatment according to PEG guidelines. Although this study is unique in combining analyses of clinical and economical outcomes in HAP/VAP according to IIAT adequacy, other studies reveal similar results. Orrick et al. performed an analysis with a very similar design for community-acquired pneumonia (CAP). In their dataset, the majority of patients $(75.8 \%)$ received preferred antibiotics according to the IDSA guidelines. The group treated with preferred antibiotics had a shorter mean length of hospital stay (4.5 vs 6.8 days, $p=0.002$ ), a lower total cost of hospitalization (median USD 2,047 vs. 3,805, $\mathrm{p}=0.021$ ) and lower antibacterial costs (median USD 97 vs. 171, $\mathrm{p}=0.038)$ compared with patients who did not receive the preferred therapy [33]. They concluded that introducing and intensifying adherence to IDSA guidelines reduces the economic burden associated with CAP.

Bird et al. performed an analysis comparing data before vs. after introduction of a guideline for a VAP prevention bundle in surgical ICUs (SICU). Prior to the introduction of the bundle, VAP was recorded at a rate of 10.2 cases per 1000 ventilator days. Compliance with the VAP bundle increased over the study period from $53 \%$ and $63 \%$ to $91 \%$ and $81 \%$ in the participating SICUs, respectively. The rate of VAP decreased to 3.4 cases per 1000 ventilator days. Estimated cost savings amounted to USD 1.08 million [34]. The authors concluded that the initiation and introduction of the bundle reduced the incidence of VAP and associated cost.

Improved survival was also shown in a retrospective analysis of CAP cases by Frei et al. They found that guideline-discordant therapy was associated with an increase in inpatient mortality ( $25 \%$ vs. $11 \%$; odds ratio $=2.99$ [95\% CI, 1.08-9.54])[35].

Ott et al. (2) investigated patients with HAP/VAP and MRSA vs. MSSA as the underlying pathogen. For MRSA patients, the cost differences were much higher than in prior studies ( $€ 60,684$ vs. $€ 38,731$; $p=0.01$ ). Moreover, more patients died in the MRSA-group. (13 vs. 1 deaths, $\mathrm{p}<0.001)$. We could not do a subgroup analysis for the MRSA patients as we did not collect microbiology results in our study.

In our study, the most frequent type of inadequate IIAT was monotherapy when the guideline recommends a combination therapy. Particularly in patients at high risk of involvement of complicated pathogens (CPRS score of $\geq 6$ ), the outcomes were significantly better when an adequate IIAT was chosen. This correlation was well established in analyses performed by Kollef et al., Kolenti et al. and other groups [8, 3638].

Possible limitations of our study include the following. While the clinical and economical resource consumption was not significantly different between the subpopulation with adequate vs. inadequate IIAT and the overall morbidity was very well comparable, we found a significant difference in the CPRS score groups $(3.5+/-2.2$ vs. $6.0+/-2.5)$. It appears that patients at higher risk of complicated pathogen involvement are more likely to receive inadequate IIAT. Another possible explanation is that experience-based local standards for IIAT are well sufficient for patients with lower CPRS score but inadequate for patients with higher CPRS score.

If a standard therapy regimen is applied to all HAP/VAP patients without applying the risk score, the number of adequately treated patients would be lower in patients with high CPRS. This is underlined by an analysis of IIAT adequacy according to the PEG therapy groups: more group I patients received adequate IIAT compared to group III patients who were more likely to receive inadequate IIAT.

However other cost drivers could have influenced the differences between the subpopulations with adequate vs. inadequate IIAT. Therefore we analysed costrelevant CPRS parameters with a significant difference of occurrence in the GA vs. NGA groups, the most prominent being 'extrapulmonary organ failure' (1 vs. 12 patients) and 'late onset' (7 vs. 19 patients). Logistic regression did not reveal any significant associations with cost, LOS or HMV.

\section{CONCLUSION}

Adequate initial intravenous antibiotic therapy of HAP/VAP according to PEG guidelines was clinically superior and cost-saving vs. inadequate IIAT. Rates of survival rates and clinical improvement were significantly higher in patients treated according to guideline recommendations. Length of stay, duration of mechanical ventilation, total costs and ICU costs were significantly lower as well for the subpopulation receiving adequate IIAT.

The most frequent type of inadequate IIAT was monotherapy in patients where the PEG guidelines recommend a combination therapy.

Moreover it appears that experience-based therapeutic strategies which are most likely in place in every hospital are mostly sufficient for patients with lower risk for complicated pathogens but not for those with a higher CPRS score. Educating physicians on guideline recommendations in patients with higher CPRS scores - ideally adapted to the individual hospital's resistance patterns - may improve treatment adequacy and thus outcomes in HAP/VAP with the add-on benefit of cost reductions. In particular, the use of combination therapies as recommended by the guidelines may help to achieve a higher rate of adequate IIAT and thus improve clinical outcomes and economic parameters.

Using the data from the German KISS surveillance program [5] one could extrapolate an yearly number of 30,090 HAP/VAP cases in German ICUs. Assuming that the observed rate of adequate IIAT of less than $50 \%$ was generalizable to all German ICUs treating HAP/VAP patients, the consistent use of IIAT according to PEG guidelines may save 2,042 lives and total costs of $€ 125,819,000$. 
Acknowledgements: We thank the participating hospitals and particularly the staff involved in providing data and medical records. The author's like to thank Dr. Béatrice Grabein from the Munich University's institute for clinical microbiology and hospital hygiene for her contributions in the planning phase of this study.

Funding: This study was supported by two unrestricted research grants from Pfizer Inc., New York, NY (USA), and Pfizer Pharma GmbH, Berlin, Germany.

\section{Conflict of interest disclosures:}

M. H. Wilke received consultancy fees and honoraria from Pfizer Pharma GmbH, Novartis Pharma Gmbh, and Wyeth Pharma GmbH (until 2009).

R. F. Grube received consultancy honoraria from Pfizer Pharma GmbH, Novartis Pharma Gmbh, and Wyeth Pharma GmbH (until 2009).

K. F. Bodmann received honoraria for lectures from Pfizer Pharma GmbH, Novartis Pharma GmbH, Bayer Pharma $\mathrm{GmbH}$, Infectopharm GmbH, Janssen-Cilag GmbH, B.R.A.H.M.S GmbH, MSD Merck, Sharp \& Dome GmbH

\section{REFERENCES}

1. DiCocco JM, Croce MA. Ventilator-associated pneumonia: an overview. Expert Opin Pharmacother. 2009 Jun;10(9):1461-7.

2. Ott E, Bange FC, Reichardt C, Graf K, Eckstein M, Schwab F, et al. Costs of nosocomial pneumonia caused by meticillin-resistant Staphylococcus aureus. J Hosp Infect. 2010 Dec;76(4):300-3.

3. Amin A. Clinical and economic consequences of ventilator-associated pneumonia. Clin Infect Dis. 2009 Aug 15;49 Suppl 1:S36-43.

4. Thompson DA, Makary MA, Dorman T, Pronovost PJ. Clinical and economic outcomes of hospital acquired pneumonia in intra-abdominal surgery patients. Ann Surg. 2006 Apr;243(4):547-52.

5. Geffers C, Gastmeier P. Nosocomial Infections and Multidrug-resistant Organisms in Germany: Epidemiological Data From KISS (The Hospital Infection Surveillance System). Dtsch Arztebl Int. 2011 Feb;108(6):87-93.

6. van der Kooi TI, Mannien J, Wille JC, van Benthem BH. Prevalence of nosocomial infections in The Netherlands, 2007-2008: results of the first four national studies. J Hosp Infect. 2010 Jul;75(3):168-72.

7. Rosenthal VD, Maki DG, Jamulitrat S, Medeiros EA, Todi SK, Gomez DY, et al. International Nosocomial Infection Control Consortium (INICC) report, data summary for 2003-2008, issued June 2009. Am J Infect Control. 2010 Mar;38(2):95-104 e2.

8. Kollef MH. Inadequate antimicrobial treatment: an important determinant of outcome for hospitalized patients. Clin Infect Dis. 2000 Sep;31 Suppl 4:S131-8.

9. Harmanci A, Harmanci O, Akova M. Hospital-acquired pneumonia: challenges and options for diagnosis and treatment. J Hosp Infect. 2002 Jul;51(3):160-7.

10. Mehta RM, Niederman MS. Nosocomial pneumonia. Curr Opin Infect Dis. 2002 Aug;15(4):387-94.

11. Hernandez G, Rico P, Diaz E, Rello J. Nosocomial lung infections in adult intensive care units. Microbes Infect. 2004 Sep;6(11):1004-14.

12. Ramphal R. Importance of adequate initial antimicrobial therapy. Chemotherapy. 2005 Jul;51(4):171-6.

13. Torres A. The new American Thoracic Society/Infectious Disease Society of North America guidelines for the management of hospital-acquired, ventilator-associated and healthcare-associated pneumonia: a current view and new complementary information. Curr Opin Crit Care. 2006 Oct;12(5):444-5.
14. Bauer TT, Lorenz J, Bodmann KF, Vogel F. [Abbreviated guidelines for prevention, diagnostics, and therapy of nosocomial pneumonia]. Med Klin (Munich). 2005 Jun 15;100(6):355-60.

15. Bodmann KF. Current guidelines for the treatment of severe pneumonia and sepsis. Chemotherapy. 2005 Aug; 51(5):227-33.

16. Niederman MS. Can guidelines for the treatment of respiratory infections lead to reduced rates of antibiotic resistance? Semin Respir Infect. 2001 Sep;16(3):203-9.

17. Rotstein C, Evans G, Born A, Grossman R, Light RB, Magder S, et al. Clinical practice guidelines for hospitalacquired pneumonia and ventilator-associated pneumonia in adults. Can J Infect Dis Med Microbiol. 2008 Jan; 19(1):19-53.

18. Martinez R, Reyes S, Lorenzo MJ, Menendez R. Impact of guidelines on outcome: the evidence. Semin Respir Crit Care Med. 2009 Apr;30(2):172-8.

19. Wilke MH, Grube R. Pharmaco-economic evaluation of antibiotic therapy strategies in DRG-based healthcare systems - a new approach. Eur J Med Res. 2010 Nov 30; 15(12):564-70.

20. Klersy C, De Silvestri A, Gabutti G, Raisaro A, Curti M, Regoli F, et al. Economic impact of remote patient monitoring: an integrated economic model derived from a meta-analysis of randomized controlled trials in heart failure. Eur J Heart Fail. 2011 Apr;13(4):450-9.

21. Pretto M, Spirig R, Kaelin R, Muri-John V, Kressig RW, Suhm N. Outcomes of elderly hip fracture patients in the Swiss healthcare system: A survey prior to the implementation of DRGs and prior to the implementation of a Geriatric Fracture Centre. Swiss Med Wkly. 2010;140(140): w13086.

22. Lotter O, Dolderer J, Stahl S, Atzei A, Haerle M, Schaller HE. Comparison of Hand Surgery in the German and Italian DRG Systems. Gesundheitswesen. 2010 Sep 30; 30: 30.

23. Gorenoi V, Dintsios CM, Schonermark MP, Hagen A. Intravascular brachytherapy for peripheral vascular disease. GMS Health Technol Assess. 2008;4(4):Doc08.

24. Wilke MH, Hocherl E, Scherer J, Janke L. [Introduction of the new DRG-based reimbursement system in German hospitals--a difficult operation? Experiences and possible solutions from the viewpoint of trauma surgery]. Unfallchirurg. 2001 May;104(5):372-9.

25. Flanders SA, Collard HR, Saint S. Nosocomial pneumonia: state of the science. Am J Infect Control. 2006 Mar; 34(2):84-93.

26. Abdel-Fattah MM. Nosocomial pneumonia: risk factors, rates and trends. East Mediterr Health J. 2008 May-Jun; 14(3):546-55.

27. Amaral SM, Cortes Ade Q, Pires FR. Nosocomial pneumonia: importance of the oral environment. J Bras Pneumol. 2009 Nov;35(11):1116-24.

28. Baughman RP. Nosocomial pneumonia: the gorilla in the ICU. J Intensive Care Med. 2003 Jul-Aug;18(4):2278.

29. Ewig S, Gillissen A. [Diagnostic and therapeutic criteria for nosocomial pneumonia]. Med Klin (Munich). 2000 Sep 15;95(9):533-4.

30. Fabian TC. Empiric therapy for pneumonia in the surgical intensive care unit. Am J Surg. 2000 Feb;179(2A Suppl):18S-23S; discussion 4S-5S.

31. Fleming CA, Balaguera HU, Craven DE. Risk factors for nosocomial pneumonia. Focus on prophylaxis. Med Clin North Am. 2001 Nov;85(6):1545-63.

32. Pletz MW, Burkhardt O, Welte T. Nosocomial methicillin-resistant Staphylococcus aureus (MRSA) pneumonia: linezolid or vancomycin? - Comparison of pharmacology and clinical efficacy. Eur J Med Res. 2010 Nov 30;15(12):507-13. 
33. Orrick JJ, Segal R, Johns TE, Russell W, Wang F, Yin DD. Resource use and cost of care for patients hospitalised with community acquired pneumonia: impact of adherence to infectious diseases society of america guidelines. Pharmacoeconomics. 2004;22(11):751-7.

34. Bird D, Zambuto A, O'Donnell C, Silva J, Korn C, Burke $\mathrm{R}$, et al. Adherence to ventilator-associated pneumonia bundle and incidence of ventilator-associated pneumonia in the surgical intensive care unit. Arch Surg. 2010 May; 145(5):465-70

35. Frei CR, Attridge RT, Mortensen EM, Restrepo MI, Yu $\mathrm{Y}$, Oramasionwu CU, et al. Guideline-concordant antibiotic use and survival among patients with community-acquired pneumonia admitted to the intensive care unit. Clin Ther. 2010 Feb;32(2):293-9.

36. Koulenti D, Rello J. Hospital-acquired pneumonia in the 21 st century: a review of existing treatment options and their impact on patient care. Expert Opin Pharmacother. 2006 Aug;7(12):1555-69.
37. Joseph NM, Sistla S, Dutta TK, Badhe AS, Parija SC. Ventilator-associated pneumonia: a review. Eur J Intern Med. 2010 Oct;21(5):360-8.

38. Kieninger AN, Lipsett PA. Hospital-acquired pneumonia: pathophysiology, diagnosis, and treatment. Surg Clin North Am. 2009 Apr;89(2):439-61, ix.

Received: May 1, 2011 / Accepted: June 10, 2011

Address for correspondence:

Dr. Michael Wilke

Dr. Wilke GmbH

inspiring.health

Joseph-Wild-Str. 13

81829 Munich

Germany

Tel.: $\quad+49(0) 8918908376-1$

Fax: $\quad$ +49(0)8918908376-9

E-mail: michael.wilke@d-w-g.de 